\title{
Delay of the reinforcing opportunity to speak in reply under invariable initial disagreement
}

\author{
ROBERT FRANK WEISS, RICHARD A. FEINBERG, \\ ROBERT E. CRAMER, and JANELLE SCHOEDEL \\ University of Oklahoma, Norman, Oklahoma 73019
}

\begin{abstract}
Speaking in reply has the functional properties of reinforcement, and delay of the opportunity to speak in reply has the functional properties of delay of reinforcement. In an experimental conversation modeled on discrete-trials instrumental conditioning, instrumental response speeds were faster when the opportunity to speak in reply was immediate rather than delayed $(\mathrm{N}=80, \mathrm{p}<.05)$. Invariable initial disagreement impairs acquisition, but not sufficiently to prevent a sizeable delay of reinforcement effect.
\end{abstract}

Participation in conversation can be reinforced by the opportunity to speak in reply to a person of differing opinion. Latency data from eight experiments show a fundamental similarity to a discrete-trials instrumental conditioning model in demonstrating replicable analogs of (a) acquisition, (b) extinction, (c) partial reinforcement effects, (d) drive effects, (e) a delay of reinforcement gradient, (f) correlated reinforcement effects, (g) correlated delay of reinforcement effects, and (h) intermittent shock effects (Weiss, Beck, \& Stich, 1972; Weiss, Boyer, Colwick, \& Moran, 1971; Weiss, Lombardo, Warren, \& Kelley, 1971; Weiss, Williams, \& Miller, 1972). The importance of studying one person's reply to another is generally acknowledged by researchers in interpersonal communication, but the reinforcing function of speaking in reply had not previously been identified and explored. If speaking in reply has the functional properties of reinforcement, then the time interval between an instrumental response and the reinforcing reply should have the functional properties of delay of reinforcement. One property of delay of reinforcement is that instrumental responses reinforced with a short delay of reinforcement are faster than responses reinforced with a long delay of reinforcement (e. g., Fowler \& Trapold, 1962; Logan, 1960; Tarpy \& Sawabini, 1974), and a corresponding result should therefore hold for speaking in reply. This prediction was confirmed in three previous experiments, which included a perfectly monotonic six-point delay of reinforcement gradient and a correlated-delay effect (Weiss, Beck, \& Stich, 1972; Weiss, Boyer, Colwick, \& Moran, 1971; Weiss, Lombardo, Warren, \& Kelley, 1971). The present experiment was designed to evaluate a methodological innovation in the general procedure of our experimental conversations, and delay was employed as an independent variable specifically because

This study was supported in part by NIMH Predoctoral Research Fellowship 1 F 31 MH05254-01 to R. Feinberg, under the sponsorship of R. Weiss. Reprints may be obtained from R. F. Weiss, Department of Psychology, University of Oklahoma, Norman, Oklahoma 73019. its effects were sufficiently determinate, replicable, and unproblematical to permit an unambiguous assessment of the efficacy of the innovation in our methodology. The innovation was intended particularly for studies in which it was desirable to have complete uniformity of disagreement or agreement among the participants in the conversation and is described in the Method section of this paper under the heading "Initial Disagreement."

\section{METHOD}

On each trial the subject listened to another person's opinion and could then press a switch (the instrumental response), the reinforcement for which was the opportunity to speak in reply to the other person.

\section{Conditioning}

The subject was seated at a table facing the control room wall, which included four one-way vision windows. Instructional signals appeared in each window upon illumination. The signals were the large printed words: (1) "listen," (2) "throw switch if you wish to comment," (3) "talk," and (4) "move dial to final opinion." A panel mounted on the table top contained the subject's "comment" switch (a telephone toggle switch with a spring return), his intercom, and a dial used in the masking task.

An experimental trial began with the "listen" signal, followed by the other person's statement. When the statement ended, the experimenter operated the control which (a) presented the CS, the signal "throw switch if you wish to comment," and (b) started the latency timer. When the subject threw the comment switch, the latency timer automatically stopped, measuring latency to $.01 \mathrm{sec}$. The "talk" signal followed the switch-press response and the subject spoke in reply. The procedure described was closely modeled on discrete-trials instrumental conditioning. The reinforcement (speaking in reply) was contingent on the instrumental response (switch pressing). The dependent variable was speed (100/latency) measured from the time of the presentation of the CS (signal "throw switch if you wish to comment") to the instrumental switch-pressing response. An interval timer automatically regulated the delay between the instrumental switch-pressing response and the onset of the reinforcing opportunity to speak in reply. Details of delay procedure follow Weiss, Boyer, Colwick, and Moran (1971). 


\section{Deception and Masking Task}

The experiment was represented to the subjects as a study of opinion change. "We are interested in how your opinion may be affected by what someone else says; how your opinion may be affected by what you yourself say; and how what you say may affect the opinions of someone else." As a masking task, after each trial the subject indicated whether he had changed his opinion by moving a dial. Questionnaire data (Weiss, Lombardo, Warren, \& Kelley, 1971) showed that the subjects believed this deception; the switch appeared to them as a mere incidental to the serious business of opinion change through conversation. The "other person," simulated by a tape recorder, was said to be in another room from the subject, and the experimenter was in fact in another room. The subject addressed the "other person" and the experimenter over an intercom system and could also hear the experimenter give instructions and occasional comments ("speak a little louder, Subject A") to the nonexistent other person.

\section{Subjects}

The subjects were 80 students from the introductory psychology pool, equally divided between 0 - and 6-sec delay groups, with counterbalanced procedures specified below.

\section{Initial Disagreement}

Our usual practice has been to begin the large majority of trials with an initial disagreement, together with a sprinkling of initial agreements. The inclusion of these initial agreements has obvious advantages for the plausibility of the experimental rationale as represented to the subjects, but certain research problems require that all experimental trials begin with either disagreement or agreement. The innovation in method employed here was to assess a uniform initial disagreement procedure employing a now well-researched independent variable, delay of the reinforcing opportunity to speak in reply.

The opinions expressed by the "other person" were selected by means of a 25 -item questionnaire administered to each subject just before the experimental session. The subjects indicated their opinions on each item and ranked the 12 items of greatest interest from among those on which they had an opinion. There was never any difficulty in identifying enough items, because the 25 items of the questionnaire had themselves been selected for interest and opinionation from a longer questionnaire administered to a sample from the same population. A magazine of 100 individual tape cassetes had been prepared, 4 for each item, male-female by pro-con, so that each subject was exposed to tapes contrary to his own opinion by another person of the same sex. The tapes were presented in randomized order. These tapes were different from the ones used in all but one of our previous studies and were intended particularly for studies in which it was desirable to have complete uniformity of disagreement or agreement, together with a structure which made it possible (together with another hundred tapes) to closely specify the strength of the argument.

\section{RESULTS AND DISCUSSION}

The meticulously prepared and implemented methodological innovation did not prove to be a methodological improvement. Our previous research has repeatedly confirmed gradual increments in response speed over the course of trials. We now report what appears to be a consistent pattern of results under conditions of uniformity of disagreement: impaired acquisition following initial differentiation among the experimental groups. This was the result obtained in our one previously reported study under conditions of uniformity of disagreement (Weiss, Williams, \& Miller, 1972), but that study employed a previously unresearched independent variable, for which purpose the uniformity of disagreement methodology was introduced. The present experiment eliminates any lingering suspicions of chance results and, by dint of the well-researched delay variable, any peculiarity of the independent variable in the previous study. In both experiments, however, the entire set of tapes was different from the tapes used in all of our previous studies. A third study (Weiss, Miller, Steigleder, \& Denton, in press), now demonstrates that trials effects under uniformity of disagreement are not due to an adventitious characteristic of particular tapes by replicating the experiment of Weiss, Williams, and Miller (1972) with well-rehearsed live confederates replacing the tapes.

A procedure which impairs acquisition is unfavorable for delay of reinforcement effects, but the short delay group was significantly faster than the long delay, tested over a block of the last six trials $[\mathrm{t}(78)=1.84, \mathrm{p}<.05]$. Since a "long" delay of $6 \mathrm{sec}$ is quite moderate by the standard of Weiss, Boyer, Colwick, and Moran's (1971) continuously decreasing delay gradient $(0,3,6,9$, $15,21 \mathrm{sec})$, these results provide a useful confirmation of the replicability of the effect of delay of the reinforcing opportunity to speak in reply.

\section{REFERENCES}

Fowler, H., \& Trapold, M. A. Escape performance as a function of delay of reinforcement. Journal of Experimental Psychology, 1962, 63, 464-467.

Logan, F. A. Incentive. New Haven: Yale University Press, 1960.

TARPY, R. M., \&.SAwabini, F. L. Reinforcement delay: A selective review of the last decade. Psychological Bulletin, 1974, 81, 984-997.

Weiss, R. F., Beck, C. M., \& Stich, M. H. Correlated delay of reinforcement in the instrumental conditioning of conversational behavior. Psychonomic Science, 1972, 28, 211-212.

Weiss, R. F., Boyer, J. L., Colwick, J. T., \& Moran, D. J. A delay of reinforcement gradient and correlated reinforcement in the instrumental conditioning of conversational behavior. Journal of Experimental Psychology, 1971, 90, 33-38.

WeISS, R. F., LOMBARDo, J. P., WARREN, D. R., \& Kelley, K. A. The reinforcing effects of speaking in reply. Journal of Personality and Social Psychology, 1971, 20, 186-199.

Weiss, R. F., Miller, F. G., Steigleder, M. K., \& Denton, D. A. Drive effects on instrumental response speed induced by intermittent disagreement in conversation. Bulletin of the Psychonomic Society, in press.

Weiss, R. F., Williams, M. J., \& Miller, C. M. Disagreementinduced drive in conversation: A social analog of intermittent shock in escape conditioning. Psychonomic Science, 1972, 29. 342-344.

(Received for publication May 20, 1976.) 